\title{
A quantitative understanding of microRNA- mediated competing endogenous RNA regulation
}

\author{
Ye Yuan ${ }^{\dagger}$, Xinying Ren ${ }^{\dagger}$, Zhen Xie and Xiaowo Wang* \\ Ministry of Education Key Laboratory of Bioinformatics and Bioinformatics Division, Center for Synthetic and Systems Biology, \\ Tsinghua National Laboratory for Information Science and Technology/Department of Automation, Tsinghua University, Beijing \\ 100084, China \\ * Correspondence: xwwang@tsinghua.edu.cn
}

Received November 23, 2015; Accepted January 3, 2016

\begin{abstract}
MicroRNA (miRNA) plays key roles in post-transcriptional regulations. Recently, a competing endogenous RNA (ceRNA) hypothesis has been proposed that miRNA targets could communicate and regulate each other through titrating shared miRNAs, which provides a new layer of gene regulation. Though a number of ceRNAs playing biological functions have been identified, the ceRNA hypothesis remains controversial. Recent experimental and theoretical studies argued that the modulation of a single RNA species could hardly change the expression level of competing miRNA targets through ceRNA effect under normal physiological conditions. Here, we reviewed a common framework to model miRNA regulations, and summarized the current theoretical and experimental studies for quantitative understanding ceRNA effect. By revisiting a coarse-grained ceRNA model, we proposed that network topology could significantly influence the competing effect and ceRNA regulation at protein level could be much stronger than that at RNA level. We also provided a conditional independent binding equation to describe miRNA relative repression on different target, which could be applied to quantify siRNA off-target effect.
\end{abstract}

Keywords: microRNA regulation; competing endogenous RNA; molecular titration; quantitative model; complex networks

\section{INTRODUCTION}

MicroRNA(miRNA) is a class of small non-coding RNAs that modulate gene expression post-transcriptionally. In animal cells, nascent miRNA transcripts (pri-miRNAs) are first processed to pre-miRNAs with the well-known stem-loop structure by the RNase enzyme Drosha [1]. Pre-miRNAs are exported to cytoplasm and further processed by another RNase enzyme Dicer to release 22 nt long mature miRNAs, which are bound by Argonaute protein (Ago) to form the RNA induced silencing complex (RISC). MiRNA could guide RISC to bind miRNA response elements (MREs) on target RNAs through complementary base pair interaction. Typically, perfect complementarity leads to target RNA cleavage, which is known as the RNA interference (RNAi) pathway [2-4], while imperfect complementarity causes target RNA destabilization and translation inhibition, which is known as the miRNA pathway $[5,6]$.

It has been well characterized that miRNA repression is dependent on the sequence characteristics. Sequence complementarity to the 5'-end 2-7 position ("seed") of the miRNA could trigger target repression $[3,7,8]$, while different base-pairing pattern leads to different repression efficiency. Dozens of computational algorithms have been developed to predict miRNA-target interactions based on

\footnotetext{
$\uparrow$ These authors contribute equally to this work.

This article is dedicated to the Special Collection of Recent Advances in Next-Generation Bioinformatics (Ed. Xuegong Zhang).
} 
sequence features $[7,9,10]$. On the other hand, recent studies suggested that the copies per cell (cpc) of one RNA species could vary from a few to tens of thousands, and the cpc of the total RNA in different type of cells could vary from $\sim 50,000$ to $\sim 500,000$ [11]. The same miRNA-target gene pairs could show dramatic difference repression efficiency under different molecular environment $[12,13]$. Incorporating expression quantities of miRNAs and their targets could allow a more accurate prediction of functional miRNA-target interactions [1416].

Due to the fact that merely 6-nt complementarity to the "seed" could induce miRNA binding, one miRNA species could simultaneously regulate multiple target RNA species, including protein-coding RNA (mRNA), small non-coding RNA, long non-coding RNA (lncRNA), pseudogene and circular RNA [17,18]. Similarly, one target RNA species could be simultaneously regulated by multiple miRNAs. In this way, miRNAs and their target RNAs compose a complex multilayered interaction network [17-19]. It has been shown that by attenuating shared miRNAs, target RNAs could crosstalk and regulate each other, and such RNAs are known as competing endogenous RNAs (ceRNAs) [17,20-23]. CeRNA regulation has been proved to play crucial roles in many biological processes including development [24] and cancer [25-28], which have been reviewed by recent papers [18,27]. Using in silico and experimental approaches, researchers have revealed that ceRNA effect is sensitive to miRNA-target relative abundance, binding energy, and the stability of miRNA and their targets [2931]. However, several recent theoretical and experimental works also argued that obvious gene expression changes due to ceRNA effect require an extreme high level of miRNA targets, which is not feasible except in some special physiological or disease status [11,31,32]. The discoveries and controversies demand a better quantitative understanding of ceRNA regulation.

In this paper, we first briefly summarized experimental approaches to quantify miRNA regulation, and then introduced a coarse-grained model to quantitatively analyze miRNA regulatory networks. Next, we reviewed recent progress towards quantitative understanding of miRNA regulation especially the ceRNA effect, and discussed the controversy debate whether RNA competing effect could induce significant gene expression changes under normal physiological environment. By revisiting the mathematic model, we proposed that network topology could significantly influence the competing effect and the strength of ceRNA regulation at protein level could be much stronger than that at RNA level, which provides new clues to understand ceRNA regulation. Finally, we concluded by discussing the challenges and future directions for elucidation of ceRNA regulations.

\section{EXPERIMENTAL APPROACHES TO QUANTIFY miRNA REGULATION}

MiRNA were found to modulate target gene expression post-transcriptionally by both translational repression and RNA destabilization. Accordingly, many biological technologies have been developed to quantify miRNA regulations at RNA or protein level respectively [33]. For example, high throughput measurements like gene expression microarray [34] and RNA sequencing $[35,36]$ have been widely used to quantify target gene changes at RNA level. Ribosome profiling technology was used to quantify translational rates changes induced by miRNA [37], and stable isotope labeling by amino acids in cell culture (SILAC) was developed to directly quantify the changes at protein level [38-40]. Methods based on Ago crosslinking and immunoprecipitation such as RNA immunoprecipitation (RIP), crosslinking and immunoprecipitation (CLIP), and crosslinking, immunoprecipitation and sequencing of hybrids (CLASH), are of special interest [41-46]. Such methods could directly identify miRNA target and quantify miRNA occupancy at each MRE, which provides important information to build computational models to predict miRNA regulatory effect in a certain cell type $[47,48]$. However, due to the complexity of endogenous miRNA-target network [22], it is very hard to perturb and quantify the regulation of a single miRNA-target pair without influencing the others. Thus in vitro experiments were used to characterize how miRNAs find, bind and regulate their targets [3]. Recently, synthetic biology has been demonstrated to be a powerful complementary approach to study the quantitative characteristics of miRNA-mediated regulation in a largely controlled manner [31,49-51].

\section{MODELLING miRNA REGULATION}

A commonly used titrimetric chemical reaction model for miRNA regulation is shown in Figure 1A. mRNA $_{i}$ transcribes into free mRNA $\left(R_{i}\right)$ at a rate of $k_{R_{i}}$. $R_{i}$ degrades at a rate of $g_{R_{i}}$, and translates into $\operatorname{protein}_{i}$ at a rate of $k_{P_{i}}$. Protein ${ }_{i}$ degrades at a rate of $g_{P_{i}}$. $R_{i}$ can be bound by the free miRNA $\left(S_{j}\right)$ to form miRNA-target complex $\left(C_{i j}\right)$ at a rate of $k_{i j+} . C_{i j}$ dissociates into $S_{j}$ and $R_{i}$ at a rate of $k_{i j-} . C_{i j}$ degrades at a rate of $g_{i j}$, and along with the degradation, the $S_{j}$ will either degrade with probability $\alpha_{i j}$ or recycle with probability $\left(1-\alpha_{i j}\right)$. miRNA $j$ gene is transcribed and processed to free miRNA $\left(S_{j}\right)$ at a rate of $k_{S_{j}}$, and degrades at a rate of $g_{S_{j}}$. The range of parameter values used in this paper were summarized in Table 1.

According to this model, the ordinary differential equations to describe the miRNA regulatory network with $N(i=1, \ldots, N)$ mRNAs regulated by $M(j=1, \ldots, M)$ miRNAs could be represented as 
A

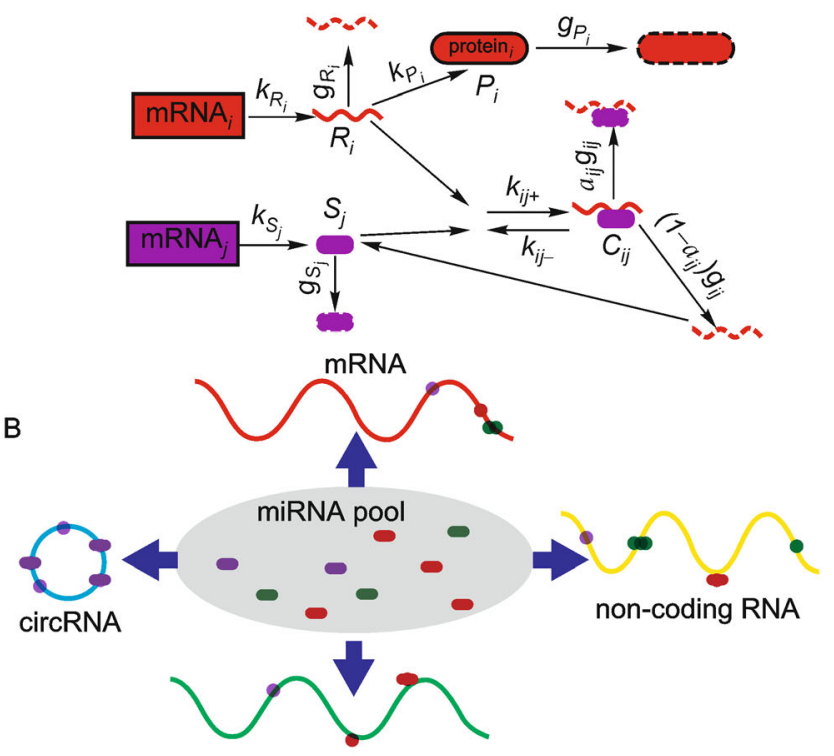

Pseudogene
C

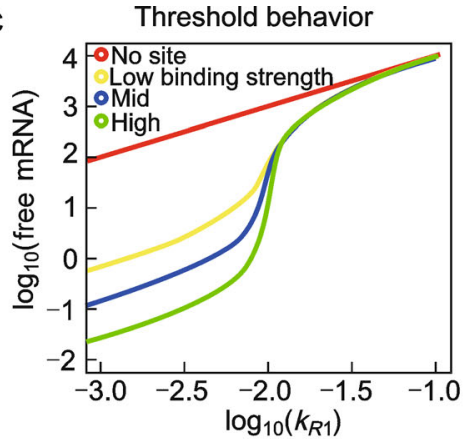

D

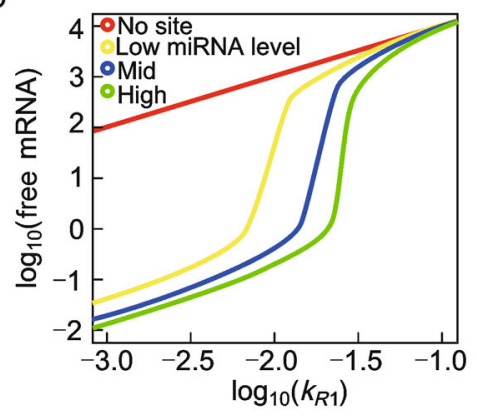

\section{miRNA level}

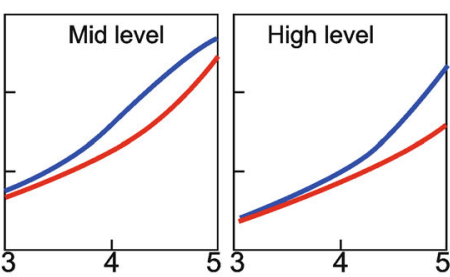

$\log _{10}$ (ceRNA1 transcriptional level)(a.u.)

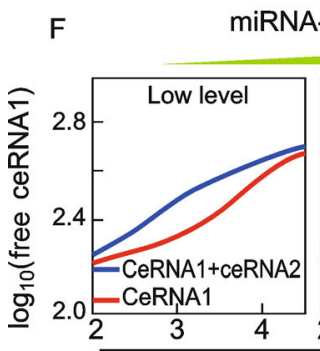

High level

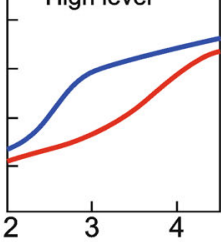

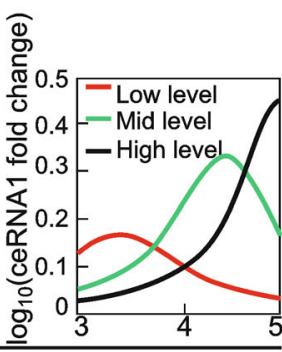

๑ิ

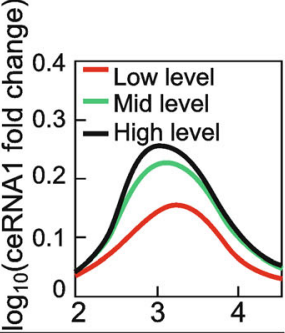

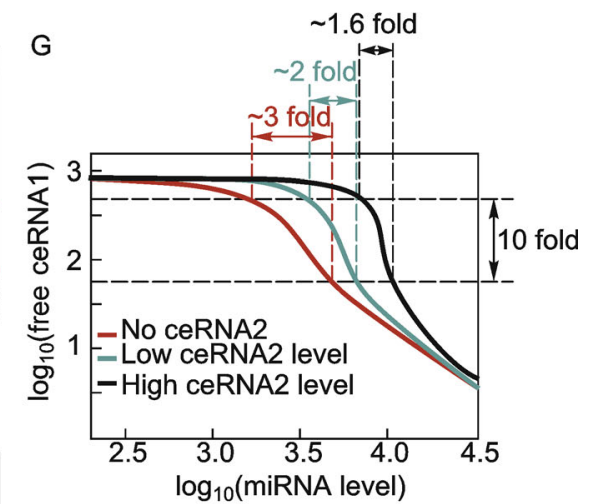

$\log _{10}($ miRNA level $)$

$\log _{10}$ (ceRNA1 transcriptional level)(a.u.)

Figure 1. Current quantitative understanding of miRNA-mediated regulation. (A) The titrimetric chemical reaction model of miRNA-induced repression upon single target RNA species. (B) MiRNAs regulate multiple RNA species. The target RNAs could be mRNA, non-coding RNA (long and short ones), pseudogene, and circular RNA. The dots on the RNAs represent MREs. (C) High miRNA binding strength sharpens the threshold-behavior of miRNA-induced repression $(M=1$, and $N=1)$ [50]. (D) MiRNA level shifts the threshold of miRNA-induced repression $(M=1$, and $N=1)$ [50]. (E) Simulated relationship between free ceRNA1 level and ceRNA1 transcriptional level with ceRNA2 containing MREs under conditions of low, middle, and high level miRNA under the condition that the transcriptional level of ceRNA2 is proportional to that of ceRNA1. Here $M=1$, and $N=1$ (red curve) or 2 (blue curve). Right panel shows the fold change of free ceRNA1 between with ceRNA2 and without ceRNA2. (F) Simulated relationship between free ceRNA1 level and ceRNA1 transcriptional level with ceRNA2 containing MREs with the low, middle, and high binding strength under the condition that the transcriptional level of ceRNA2 is proportional to that of ceRNA1 $(M=1$, and $N=1$ or 2$)$. MiRNA expression level is equal in each panel. Right panel shows the fold change of free ceRNA1 between the case with ceRNA2 (blue line) and the case without ceRNA2 (red line). (G) Simulated relationship between free ceRNA1 and miRNA transcriptional level with ceRNA2 containing MREs of no, low, high expression level $(M=1$, and $N=1$ or 2$)$. The fold changes of miRNA level needed to cause a ten-fold change of free ceRNA1 are marked. $(E),(F)$ and $(G)$ were modified from our previous work [31]. 
Table 1. Model parameter estimation.

\begin{tabular}{lll}
\hline Parameters & Value & Refs. \\
\hline Cell volume & $2000 \mu \mathrm{m}^{3}$ & {$[52]$} \\
$k_{R}, k_{S}$ & Variable & {$[53]$} \\
$g_{R}, g_{S}$ & $\sim 2.1 \times 10^{-5} \mathrm{~s}^{-1}$ & {$[53]$} \\
$\alpha$ & $0 \sim 1$ & $\mathrm{NA}$ \\
$k_{+}$ & $\sim 4.3 \times 10^{-5} \mathrm{~s}^{-1}$ & {$[3]$} \\
$k_{-}$ & $\sim 4.6 \times 10^{-4} \mathrm{~s}^{-1}$ & {$[3]$} \\
$g$ & Variable & NA \\
\hline
\end{tabular}

$$
\begin{gathered}
\frac{d S_{j}}{d t}=k_{S_{j}}-S_{j} g_{S_{j}}+\sum_{i=1}^{N}\left(-S_{j} k_{i j+} R_{i}+k_{i j-} C_{i j}+\left(1-\alpha_{i j}\right) C_{i j} g_{i j}\right), \\
\frac{d R_{i}}{d t}=k_{R_{i}}-g_{R_{i}} R_{i}+\sum_{j=1}^{M}\left(-R_{i} k_{i j+} S_{j}+k_{i j-} C_{i j}\right), \\
\frac{d C_{i j}}{d t}=k_{i j+} R_{i} S_{j}-k_{i j-} C_{i j}-g_{i j} C_{i j}, \\
\frac{d P_{i}}{d t}=R_{i} k_{p_{i}}-P_{i} g_{P_{i}} .
\end{gathered}
$$

The model could also be applied to describe miRNA-noncoding RNA, miRNA-pseudogene and miRNA-circRNA kinetic processes by setting target translation rate to zero (Figure 1B). Such model framework was first proposed to study small RNA repression in bacteria [49] and protein titration in Drosophila [54]. Mukherji et al. used a simplified model to study the miRNA-induced repression in mammalian cells [50]. Ala et al. further extended the model to study ceRNA titration effect and introduced the recycle rate of miRNA to describe catalyticity of reactions [55]. This coarse-grained model simplified the detailed biological process such as the transcription, processing and exporting process of miRNAs and mRNAs, and assumed that Ago supply is not saturated [42,56,57]. However it should be kept in mind that though the expression of Ago could reach up to $170,000 \mathrm{cpc}$ [57], Ago shortage may bias the model prediction when miRNA level is extremely high [50].

\section{QUANTITATIVE CHARACTERISTICS OF MIRNA-INDUCED REPRESSION}

The most significant quantitative property of miRNAinduced repression is the threshold-like behavior of target translational level versus transcriptional level (Figure 1C and 1D). In the condition of a constant miRNA transcriptional level, the translational level of target is close to zero if its transcriptional level is lower than the threshold, while the translational level positively correlates with the transcriptional level if it is higher than this threshold. Such threshold behavior was first found through the study of small RNA regulation in bacteria
[49], then validated for miRNA in mammalian cells [50]. Several factors impacting the threshold behavior were also proposed: the sequence complementary pattern and density of MREs that one target RNA harbors would modulate the sharpness of the threshold (Figure 1C); the concentration of miRNA would shift the threshold position (Figure 1D) [49,50].

Another interesting quantitative characteristic is that miRNA can control the protein expression noise of their target genes. Using fluctuation dissipation theory and single cell assays, Schmiedel et al. showed that miRNA can decrease the noise of low-level expressed targets, but increase the noise of high-level expressed targets [51]. Riba et al. also showed that miRNA regulation combining with transcriptional factor (TF) regulation could improve the stability of target gene expression [58]. These works imply the key role of miRNA in system stability.

\section{QUANTITATIVE CHARACTERISTICS OF miRNA-MEDIATED ceRNA REGULATION}

The concept of ceRNA regulation in mammalian cells was first proposed in 2011 [17]. The cancer repressor gene $P T E N$ was found to be regulated by several genes $[21,23]$ and its pseudogene, PTENP1 [59] through the competition for shared miRNAs. The long noncoding RNA, MD1 was also found to control muscle differentiation by regulating gene $M E F 2 C$ and $M A M L 1$ through the competing effect [20]. A large-scale extensive ceRNA network, consisting of $\sim 7,000$ genes (nodes) and $\sim 248,000$ potential ceRNA regulations (edges) was constructed by integration of miRNA-target predictions and mutual information inferred from hundreds of high throughput gene and miRNA expression profiles of glioblastoma from the Cancer Genome Atlas (TCGA) [22]. The authors proposed that the strong ceRNA regulation tends to occur in the core part of the complex ceRNA network, where nodes have high degrees. From then on, a number of functional ceRNA pairs have been discovered in diverse biological processes, especially in cancer, which has been summarized by a recent review [27].

Due to its competing nature, ceRNA regulation is highly quantitative dependent. Ala et al. built a minimal ceRNA model that two ceRNA species were regulated by one miRNA species [55]. By simulating the model, they found a threshold-like behavior in ceRNA regulation, that the ceRNA regulation only occurs near an equimolar state between miRNA and its target RNAs. They also found an asymmetric ceRNA regulation between ceRNAs at different expression level. Highly expressed ceRNA effectively regulates the low-level one, while low-level ceRNA only slightly influences the high-level one. Figliuzzi et al. proposed a mathematic definition of 
ceRNA crosstalk strength based on sensitivity analysis [60], and they stated that specific ceRNA pairs with proper characteristics could interact through selective channel of communication in the complex regulation network. Nitzan et al. found that the strength of ceRNA regulation was determined by many factors, such as generation and degradation rates of miRNA and target RNAs, and the topological distance between ceRNAs in miRNA-ceRNA network [61]. Using stochastic simulation, Ala et al. found that the indirect crosstalk in miRNAceRNA network can amplify the ceRNA regulation [55]. Combining stochastic simulation and differential equations, Noorbakhsh et al. proposed that the noise level of target genes could be a useful indicator to identify ceRNA regulation in a complex network [62].

In our previous work [31], by implementing multifluorescent synthetic gene circuits in human embryonic kidney (HEK) $293 \mathrm{H}$ cells, we validated that miRNAtarget relative abundance, hybridization free energy and the number of MREs harbored in the target RNAs could impact the strength of ceRNA regulation (Figure 1E and $1 \mathrm{~F})$. Especially, we proposed that by sequestering free miRNAs, competing RNAs could sharpen the switch-like miRNA-induced repression profoundly (Figure 1G). We also analyzed the competing effect between miRNA pathway and RNAi pathway by analyzing a special ceRNA system containing both perfectly complementary targets and imperfectly complementary targets (Figure $2 \mathrm{~A}$ ). We proposed that RNAi pathway has much lower miRNA loss rate than the miRNA pathway, which induced a nonreciprocal ceRNA effect.

\section{CONTROVERSY IN CURRENT CERNA STUDIES}

Along with rapid development of quantitative analysis, a growing number of studies have reported experimentally validated functional ceRNA pairs [63], such as PTEN and PTENP1 [20,64], PTEN and VAPA [23], PTEN and ZEB2 [21], CD44 and its ceRNAs [65,66], HMGA2 and its ceRNAs [25], lncRNA FER1L4 and its ceRNAs [67].

However, in a recent work by Denzler et al., the authors transfected specialized ceRNA (AldoA) containing designed miR-122 MREs into mouse hepatocytes, and used RNA-seq to detect the fold change of endogenous miR-122 targets caused by ceRNA competition [32]. They found that the distinct derepression of endogenous miR-122 targets only occurs when more than $100,000 \mathrm{cpc}$ AldoA RNA were transfected, which is much higher than typical endogenous gene expression level. In another theoretical work, Jens et al. used a simplified equilibrium steady state model considering miRNA-target binding energy to analyze miR-20a targets [11], and got a similar conclusion that the strong upregulation of a single mRNA species could not notably affect gene expression through ceRNA effect under most physiological conditions. The RNA-seq data of our synthetic circuit experiment [31] also indicated that only high-abundance ceRNAs can generate global derepression changes at RNA level.

However, a recent single cell reporter assay by Bosson et al. implied that several ceRNAs of only $300-1,000 \mathrm{cpc}$ could significantly derepress target genes. The authors proposed a hierarchical affinity model of miRNA target competition [29] where high-affinity targets in a low miRNA: target ratio pool were susceptible to ceRNA regulation, while low-affinity targets in a high miRNA: target ratio pool were insensitive to ceRNA regulation. Another recent experimental work further proposed that RISC availability could modulate ceRNA regulation [30]. To summarize, a number of functional ceRNA pairs have been experimentally identified, but the controversy among theories and experiments needs further investigation.

\section{REVISIT THE ceRNA MODEL}

Considering the current understanding and controversy of ceRNA regultion, we thought it is necessary to revisit the theoretical model in more details. Here, based on simulation analysis of the mathematical model we given new speculations on ceRNA regulation, which would complement current understanding of ceRNA effect.

Based on Figure 2A, we first introduce the minimum ceRNA network kinetic model in which one miRNA species regulates two target species $[31,55]$. The model details are as follow.

$$
\begin{gathered}
\frac{d S}{d t}=k_{S}-S g_{S}+\sum_{i=1}^{N}\left(-S k_{i+} R_{i}+k_{i-} C_{i}+\left(1-\alpha_{i}\right) C_{i} g_{i}\right) \\
\frac{d R_{i}}{d t}=k_{R_{i}}-g_{R_{i}} R_{i}-R_{i} k_{i+} S+k_{i-} C_{i}, \\
\frac{d C_{i}}{d t}=k_{i+} R_{i} S-k_{i-} C_{i}-g_{i} C_{i}, \\
\frac{d P_{i}}{d t}=R_{i} k_{p_{i}}-P_{i} g_{P_{i}}, \\
i=\{1,2\}, N=2 .
\end{gathered}
$$

\section{Conditional independent equation for ceRNA regulation}

By solving Equations (2) and (3) at the steady sate, we got the ratio of free RNA to the total RNA ("free ratio" for short) of each target RNA species as: 


$$
\frac{R_{1}}{R_{1}^{0}}=\frac{1}{1+O_{1} \times S}, \frac{R_{2}}{R_{2}^{0}}=\frac{1}{1+O_{2} \times S},
$$

where

$$
O_{1}=\frac{k_{1+}}{g_{R_{1}}} \times \frac{1}{1+\frac{k_{1-}}{g_{1}}}, O_{2}=\frac{k_{2+}}{g_{R_{2}}} \times \frac{1}{1+\frac{k_{2-}}{g_{2}}} .
$$

It indicates that the free ratio of one target RNA species is determined only by the free miRNA concentration $(S)$ and by the chemical reaction parameters between miRNA and this target RNA $(O)$. In another words, for ceRNAs sharing one miRNA species, the free ratio of each ceRNA is conditional independent of each other given the free miRNA concentration. Thus the free miRNA concentration is the communication channel between ceRNA1 and ceRNA2. We introduce relative repression curve as shown in Figure $2 \mathrm{~B}$ and $2 \mathrm{C}$ to represent the relative repression efficacy on ceRNA1 and ceRNA2. Each point on the curve corresponds to the free ratios of the two targets given a certain free miRNA level $(S)$. It should be noticed that the shape of the curve is determined only by parameter $O_{1}$ and $O_{2}$ (Figure $2 \mathrm{~B}$ ), rather than the expression level of miRNA or targets (Figure 2C).

Such representation could be useful to study siRNA off-target effect. In our previous work [31], we used ceRNA1 with partial pairing MRE to mimic off-target [68], and ceRNA2 with perfectly complementary MRE to mimic siRNA target (Figure 2A). By both in silico simulation and experimental validation, we demonstrated that the expression level of off-target has no impact on the shape of the relative repression curve, while the relative siRNA binding strength to target and off-target could significantly change the curve.

This computational frame work could be generalized to describe a general condition, in which one siRNA species represses one target and multiple off-targets. Interestingly, the equations still keep the form as:

$$
\frac{R_{i}}{R_{i}^{0}}=\frac{1}{1+O_{i} \times S},
$$

where,

A

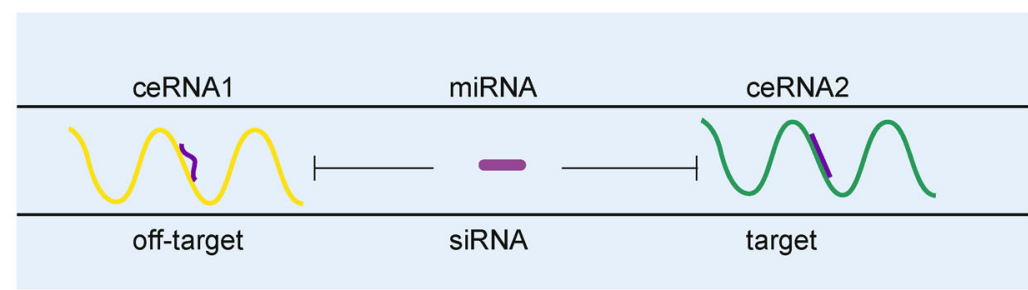

Relative repression curve
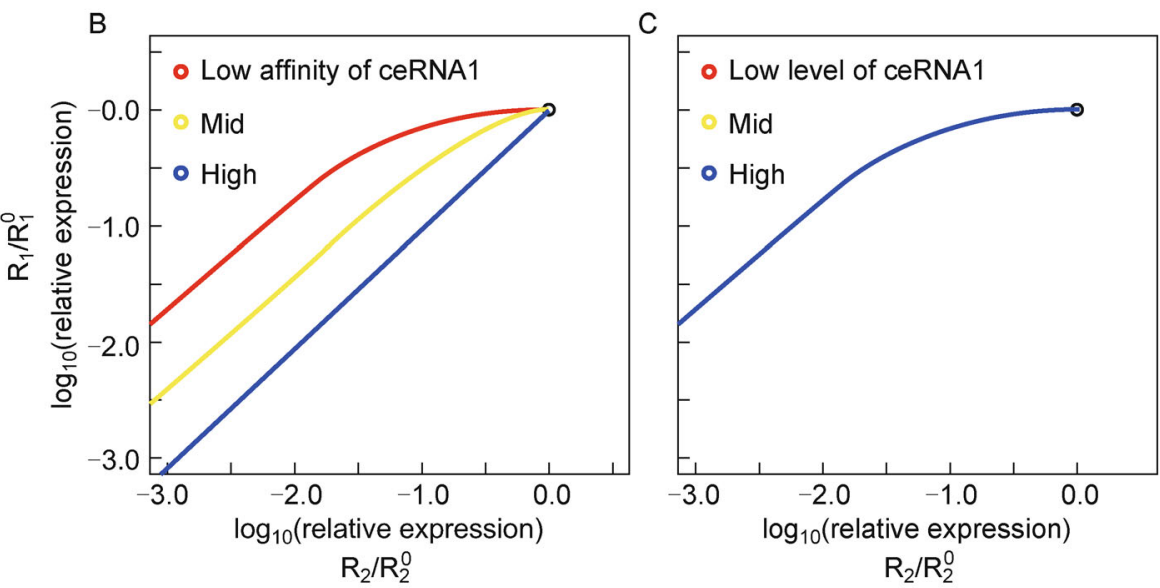

Figure 2. Relative repression curve. (A) Schematic diagram of model-guided quantitative analysis of a special ceRNA system (RNAi). CeRNA2 complementary perfectly to miRNA mimics RNAi target, while ceRNA1 complementary imperfectly to miRNA mimics off-target. (B) Binding strength between target RNA and miRNA impacts the on-target-off-target repression curve. The $x$-axis and $y$-axis are the logarithm of free ratio of the two targets respectively. (C) Expression level of off-target has no impact on the ontarget-off-target repression curve. The parameters of (B) and (C) were set as follow: $k_{R_{1}}=\{5,10,15\} \times 10^{-3}, g_{R_{1}}=1 \times 10^{-5}, g_{1}=8 \times$ $10^{-5}, a_{1}=0.5, k_{1-}=5 \times 10^{-5}, k_{1+}=\{5,10,15\} \times 10^{-5} ; \quad k_{R_{2}}=5 \times 10^{-3}, g_{R_{2}}=1 \times 10^{-5}, g_{2}=8 \times 10^{-5}, a_{2}=0.1, k_{2-}=5 \times 10^{-5}$, $k_{2+}=100 \times 10^{-5} ; g_{s}=1 \times 10^{5}, k_{s}=10^{5} \sim 10^{-1}$. 


$$
O_{i}=\frac{k_{i+}}{g_{R_{i}}} \times \frac{1}{1+\frac{k_{i-}}{g_{i}}} .
$$

Again, the shape of relative repression curve of any target-off-target pair is independent of other off-targets. Using this model, we could evaluate the influence on each off-target respectively. In general, the off-targets with low self-degradation rate $\left(g_{R_{i}}\right)$, high complex-degradation rate $\left(g_{i}\right)$ and high binding affinity $\left(k_{i+}\right)$ should be avoided when designing siRNAs.

\section{Comparison of ceRNA effect at the RNA level and the protein level of target genes}

Current literature did not carefully distinguish the ceRNA derepression effect at the RNA level from that at the protein level. For example Denzler et al. [32] concluded that ceRNA induced derepression was weak based on RNA-seq data, while recent experimental evidence supporting ceRNA hypothesis by Bosson et al. was based on protein level data (fluorescent values) [29]. It is known that RNA level could only explain part of the variability in protein levels $[53,69]$. Considering that RNA-seq data contains both free and miRNA-bond target RNAs while proteins are dominantly translated from free mRNAs [37], we speculate that the ceRNA induced derepression detected from RNA-seq data may be different from that at protein level.

We used the minimal model to simulate the strength of ceRNA-induced derepression at both RNA and protein level (Figure 3A). Here we assume that only free mRNAs could be used to translate protein with a constant rate $k_{P}$, thus the protein level is proportional to the free mRNA level at steady state. We increased the transcriptional rate of ceRNA1 $\left(k_{R_{1}}\right)$, and then measured the free (proportional to protein level) and total mRNA level of ceRNA2. As shown in Figure 3B and 3C, the strength of ceRNA regulation detected at RNA level is significantly weaker than that in protein level. To make it clearer, we marked the fold changes of free ceRNA2 and of whole ceRNA2 level caused by 10 -fold increase of $k_{R_{1}}$. In this ideal case, the protein level increases around $\sim 200$ fold, while the RNA level only increases $\sim 6$ fold (Figure 3B). Furthermore, when the self-degradation rate of ceRNA2 is larger than the degradation rate of ceRNA2 caused by miRNA, opposite trends occur (Figure 3C): along with the increase of $k_{R_{1}}$, the protein level of ceRNA2 increases while the whole ceRNA2 level decreases instead. In summary, we speculate that competing RNAs might be able to significantly modulate target gene expression at protein level with less changes at RNA level in appropriate conditions.

\section{Network topology can either enhance or diminish ceRNA effect}

Previous studies have found that network topology can impact ceRNA regulation. Nitzan et al. proposed that the long topological distance between ceRNAs weakens the ceRNA regulation strength [61]. Using stochastic simulation, Ala et al. proved that indirect ceRNA regulation through the third ceRNA species could enhance the ceRNA regulation [55]. Detailed quantitative study for ceRNA network is very hard due to the exponential growth of the complexity along with the size of network. To demonstrate the opposite effect that the network topology could bring to ceRNA regulation, we expanded the minimal model to slightly more complex structures as shown in Figure 4A and 4B. In Figure 4A, we introduced ceRNA3 to buffer the changes of free miRNA. The fold change of free ceRNA2 caused by 10 fold $k_{R_{1}}$ changes was less than 10 fold (Figure 4C), compared with the $\sim 200$ fold in Figure 3B. But when we introduced indirect regulation through ceRNA3, as shown in Figure 4B, the 10 fold $k_{R_{1}}$ changes could induce $\sim 300$ fold change of free ceRNA2, which was 1.5 fold stronger than that in Figure 3B. Thus we conclude that different network topology can either enhance or diminish ceRNA effect, and how to evaluate the topological effect is of vital importance to understand the ceRNA regulation among the complex miRNA-target network.

\section{CONCLUDING REMARKS AND FUTURE PERSPECTIVES}

MiRNA plays key roles in many biological processes. Here we focus on the quantitative characteristics of miRNA-mediated post-transcriptional regulations, especially on that of competing endogenous RNA effect. We summarized recent experimental and theoretical progresses to understand the quantitative characteristics of ceRNA regulation.

Though a number of ceRNA pairs have been discovered, a debate about ceRNA hypothesis still exists that strong upregulation of a single mRNA species may not notably affect gene regulation through ceRNA effect in normal physiological RNA molecular concentration. Based on simulation analysis, we speculated that the controversy debate might result from the incomprehension of the complex multilayered ceRNA regulation network. We showed that different network topology could either diminish or enhance the competing effect, and derepression magnitude evaluated at RNA level could be very different from that at protein level. More importantly, what we concern most is not the changes in gene expression, but the phenotypes yielded by the changes. 
A
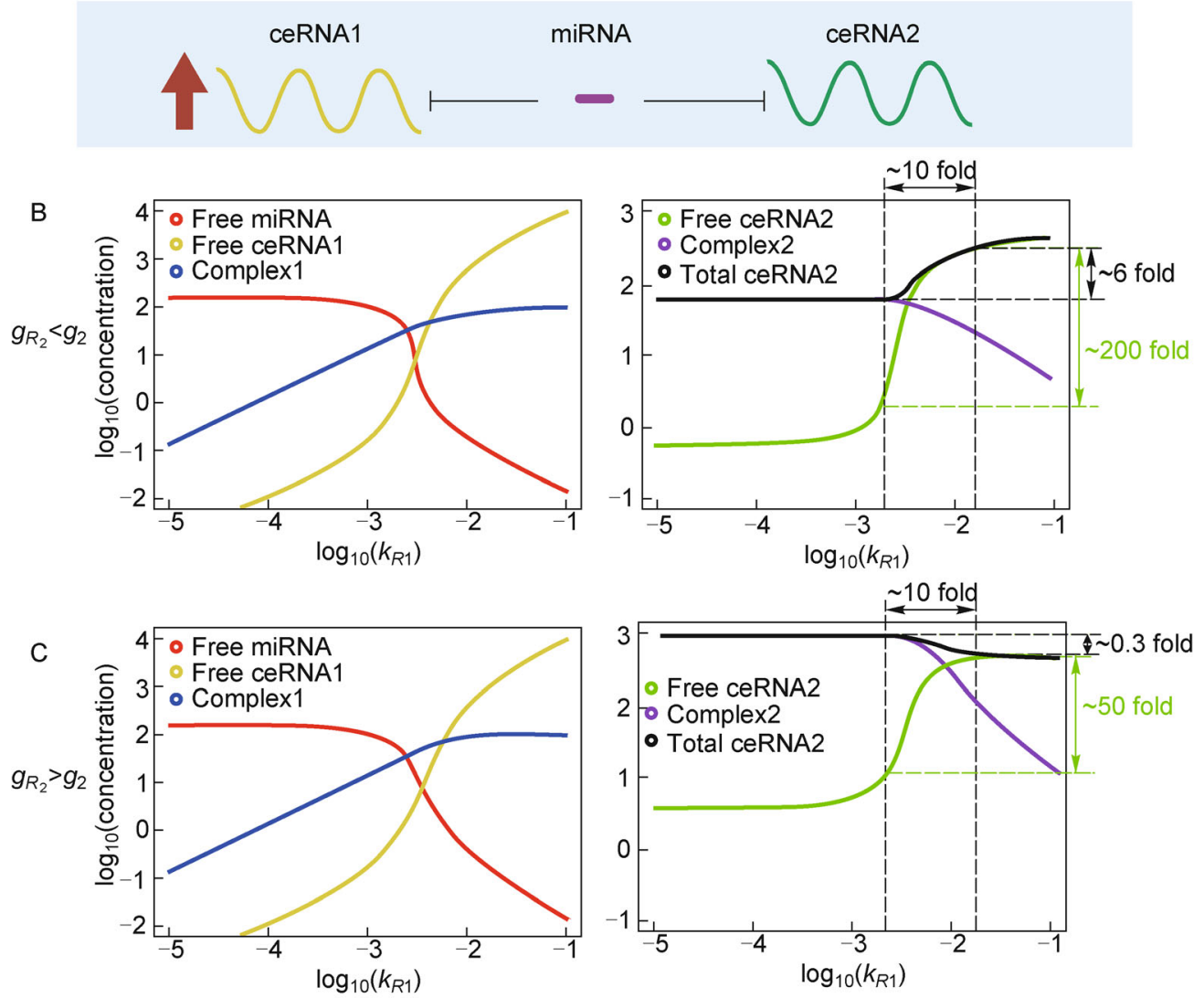

Figure 3. The difference of ceRNA regulation strength between at RNA level and at protein level. (A) Schematic diagram of the strategy of measuring the ceRNA regulation in the minimal ceRNA regulation model. We increase the transcriptional rate of mRNA1, and measure the levels of mRNA2, including the free mRNA2 that is proportional to protein level, the complex2 containing mRNA2 and miRNA and the whole mRNA2 equaling free mRNA2 plus complex2. (B) Steady-state concentrations as function of $k_{R_{1}}$ changes under the condition that $g_{R_{2}}<g_{2}$. (C) Steady-state concentrations as function of $k_{R_{1}}$ changes under the condition that $g_{R_{2}}>g_{2}$. (B) and (C), the left panel shows the steady-state concentrations of mRNA1 and miRNA. The right panel shows the steadystate concentrations of mRNA2. The fold changes of mRNAs concentrations caused by a ten-fold change of $k_{R_{1}}$ are marked. The parameters of (B) were set: $k_{R_{1}}=10^{-5} \sim 10^{-1}, g_{R_{1}}=1 \times 10^{-5}, g_{1}=8 \times 10^{-5}, \alpha_{1}=0.5, k_{1-}=5 \times 10^{-5}, k_{1+}=10 \times 10^{-5} ; k_{R_{2}}=5 \times 10^{-3}$, $g_{R_{2}}=1 \times 10^{-5}, g_{2}=8 \times 10^{-5}, \alpha_{2}=0.5, k_{2-}=5 \times 10^{-5}, k_{2+}=10 \times 10^{-5} ; g_{S}=1 \times 10^{-5}, k_{S}=4 \times 10^{-3}$. The parameters of (C) were set the same as these of (B) with an exception that $g_{2}=0.5 \times 10^{-5}$.

Therefore, it is of vital importance to uncover the quantitative relationship between the changes at gene expression level and the phenotype caused by the changes, as small differences in gene expression could yield distinct phenotypes [70]. Thus a new theoretical framework taking these aspects into account is essential to reconcile the controversy.

Predicting functional ceRNA pairs in a specific cell type at genome scale is a challenging task. Current methods detecting ceRNA pairs are mainly based on the similar work flow [22,71-73]: Firstly, estimate gene-gene association based on a large number of high throughput gene expression profiles to identify highly correlated gene pairs; secondly, filter the gene pairs using predicted or experimentally validated miRNA-target RNA pairs to predict potential ceRNA pairs; thirdly, validate the biological relevance of the predicted pairs. The major drawback of such a framework is that it requires a large sample size thus could hardly be applied to datasets with only a few samples. However, integration of multiple types of omics data may offer information for ceRNA prediction. For example, combination of mRNA-seq, miRNA-seq and Ago-HITS-CLIP data could be used to predict the target RNA's occupancy rate by each miRNA [16], while ribosome profiling and SILAC data offers the production, degradation rate and cpc of mRNA and protein [53], which could be used to validate the calculated occupancy rate. Based on the multi-level 

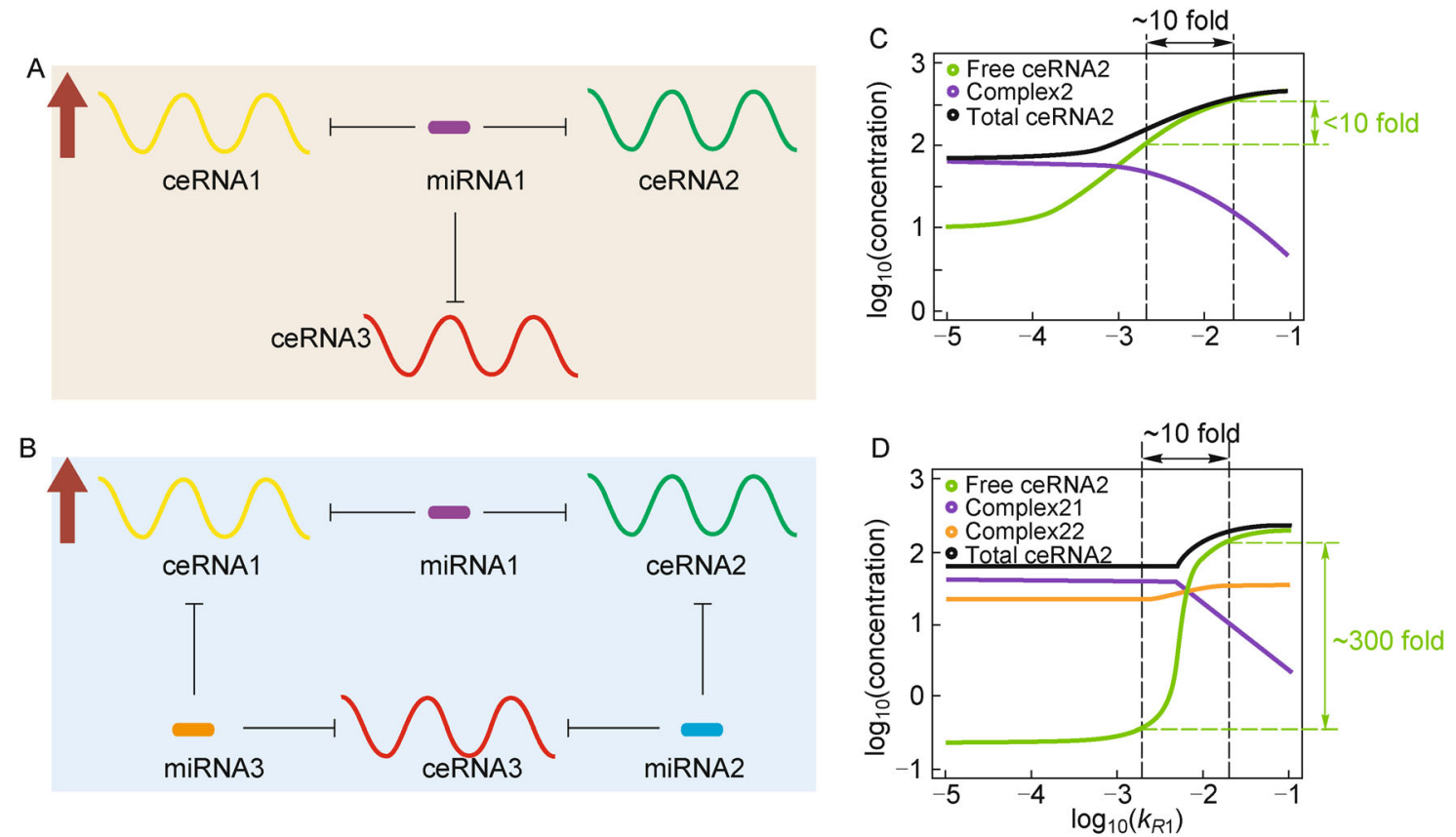

Figure 4. Network topology influences ceRNA regulation strength. (A) One miRNA species represses three ceRNA species. (B) An indirect ceRNA regulation through ceRNA3, from ceRNA1 upon ceRNA2 is introduced. (C) Steady-state concentration as a function of $k_{R_{1}}$ of the network in (A) under the condition that $g_{R_{2}}<g_{2}$. (D) Steady-state concentration as a function of $k_{R_{1}}$ of the network in (B) under the condition that $g_{R_{2}}<g_{2}$. (C) and (D), the fold changes of concentrations of ceRNA2 caused by a ten-fold change of $k_{R_{1}}$ are marked on the plots. The parameters of (C) were set: $k_{R_{1}}=10^{-5} \sim 10^{-1}, g_{R_{1}}=1 \times 10^{-5}, g_{11}=8 \times 10^{-5}, \alpha_{11}=0.5, k_{11-}=5 \times 10^{-5}$, $k_{11+}=10 \times 10^{-5} ; k_{R_{2}}=5 \times 10^{-3}, g_{R_{2}}=1 \times 10^{-5}, g_{21}=8 \times 10^{-5}, \alpha_{21}=0.5, k_{21-}=5 \times 10^{-5}, k_{21+}=10 \times 10^{-5} ; k_{R_{3}}=5 \times 10^{-3}, g_{R_{3}}=1 \times$ $10^{-5}, g_{31}=8 \times 10^{-5}, \alpha_{31}=0.5, k_{31-}=5 \times 10^{-5}, k_{31+}=10 \times 10^{-5} ; k_{S_{1}}=4 \times 10^{-3}, g_{S_{1}}=1 \times 10^{-5}$. The parameters of (D) were set: $k_{R_{1}}=10^{-5} \sim 10^{-1}, g_{R_{1}}=1 \times 10^{-5}, g_{11}=8 \times 10^{-5}, \alpha_{11}=0.5, k_{11-}=5 \times 10^{-5}, k_{11+}=10 \times 10^{-5} ; g_{13}=8 \times 10^{-5}, \alpha_{13}=0.5, k_{13-}=5 \times 10^{-5}$, $k_{13+}=10 \times 10^{-5} ; k_{R_{2}}=5 \times 10^{-3}, g_{R_{2}}=1 \times 10^{-5}, g_{21}=8 \times 10^{-5}, \alpha_{21}=0.5, k_{21-}=5 \times 10^{-5}, k_{21+}=10 \times 10^{-5} ; g_{22}=8 \times 10^{-5}, \alpha_{22}=0.5$, $k_{22-}=5 \times 10^{-5}, k_{22+}=100 \times 10^{-5} ; k_{R_{3}}=2 \times 10^{-3}, g_{R_{3}}=1 \times 10^{-5}, g_{32}=8 \times 10^{-5}, \alpha_{32}=0.5, k_{32-}=5 \times 10^{-5}, k_{32+}=100 \times 10^{-5} ; g_{33}=8$ $\times 10^{-5}, \alpha_{33}=0.5, k_{33-}=5 \times 10^{-5}, k_{33+}=100 \times 10^{-5} ; k_{S_{1}}=4 \times 10^{-3}, g_{S_{1}}=1 \times 10^{-5}, k_{S_{2}}=2 \times 10^{-3}, g_{S_{2}}=1 \times 10^{-5}, k_{S_{3}}=2 \times 10^{-3}, g_{S_{3}}$ $=1 \times 10^{-5}$

omics data from a specific cell-type, one may estimate whether the target RNA is near the threshold where it is more susceptive to the ceRNA effect. Thus, we speculate that new computational tools integrating a variety of data types may be a powerful approach to predict cell-type specific ceRNA pairs more precisely.

\section{ACKNOWLEDMENTS}

This work was supported by the National Basic Research Program (No. 2012CB316503), the National Natural Science Foundation (Nos. 61322310 and 31371341), Tsinghua University Initiative Scientific Research Program, and Tsinghua National Laboratory for Information Science and Technology.

\section{COMPLIANCE WITH EITHICS GUIDELINES}

The authors Ye Yuan, Xinying Ren, Zhen Xie and Xiaowo Wang declare that they have no conflict of interest.
This article does not contain any studies with human or animal subjects performed by any of the authors.

\section{REFERENCES}

1. He, L. and Hannon, G. J. (2004) MicroRNAs: small RNAs with a big role in gene regulation. Nat. Rev. Genet., 5, 522-531

2. Fire, A., Xu, S., Montgomery, M. K., Kostas, S. A., Driver, S. E. and Mello, C. C. (1998) Potent and specific genetic interference by doublestranded RNA in Caenorhabditis elegans. Nature, 391, 806-811

3. Wee, L. M., Flores-Jasso, C. F., Salomon, W. E. and Zamore, P. D. (2012) Argonaute divides its RNA guide into domains with distinct functions and RNA-binding properties. Cell, 151, 1055-1067

4. Zamore, P. D., Tuschl, T., Sharp, P. A. and Bartel, D. P. (2000) RNAi: double-stranded RNA directs the ATP-dependent cleavage of mRNA at 21 to 23 nucleotide intervals. Cell, 101, 25-33

5. Bartel, D. P. (2009) MicroRNAs: target recognition and regulatory functions. Cell, 136, 215-233

6. Hutvágner, G. and Zamore, P. D. (2002) A microRNA in a multiple- 
turnover RNAi enzyme complex. Science, 297, 2056-2060

7. Agarwal, V., Bell, G. W., Nam, J. W. and Bartel, D. P. (2015) Predicting effective microRNA target sites in mammalian mRNAs. Elife, 4, 4

8. Krek, A., Grün, D., Poy, M. N., Wolf, R., Rosenberg, L., Epstein, E. J., MacMenamin, P., da Piedade, I., Gunsalus, K. C., Stoffel, M., et al. (2005) Combinatorial microRNA target predictions. Nat. Genet., 37, 495-500

9. Kertesz, M., Iovino, N., Unnerstall, U., Gaul, U. and Segal, E. (2007) The role of site accessibility in microRNA target recognition. Nat. Genet., 39, 1278-1284

10. Lewis, B. P., Burge, C. B. and Bartel, D. P. (2005) Conserved seed pairing, often flanked by adenosines, indicates that thousands of human genes are microRNA targets. Cell, 120, 15-20

11. Jens, M. and Rajewsky, N. (2015) Competition between target sites of regulators shapes post-transcriptional gene regulation. Nat. Rev. Genet., $16,113-126$

12. Allantaz, F., Cheng, D. T., Bergauer, T., Ravindran, P., Rossier, M. F., Ebeling, M., Badi, L., Reis, B., Bitter, H., D’Asaro, M., et al. (2012) Expression profiling of human immune cell subsets identifies miRNAmRNA regulatory relationships correlated with cell type specific expression. PLoS One, 7, e29979

13. Cajigas, I. J., Tushev, G., Will, T. J., tom Dieck, S., Fuerst, N. and Schuman, E. M. (2012) The local transcriptome in the synaptic neuropil revealed by deep sequencing and high-resolution imaging. Neuron, 74, 453-466

14. Gennarino, V. A., Sardiello, M., Avellino, R., Meola, N., Maselli, V., Anand, S., Cutillo, L., Ballabio, A. and Banfi, S. (2009) MicroRNA target prediction by expression analysis of host genes. Genome Res. $19,481-490$

15. Jung, D., Kim, B., Freishtat, R. J., Giri, M., Hoffman, E. and Seo, J. (2015) miRTarVis: an interactive visual analysis tool for microRNAmRNA expression profile data. BMC Proc, 9, S2

16. Xie, P., Liu, Y., Li, Y., Zhang, M. Q. and Wang, X. (2014) MIROR: a method for cell-type specific microRNA occupancy rate prediction. Mol Biosyst, 10, 1377-1384

17. Salmena, L., Poliseno, L., Tay, Y., Kats, L. and Pandolfi, P. P. (2011) A ceRNA hypothesis: the Rosetta Stone of a hidden RNA language? Cell, 146, 353-358

18. Tay, Y., Rinn, J. and Pandolfi, P. P. (2014) The multilayered complexity of ceRNA crosstalk and competition. Nature, 505, 344-352

19. Taulli, R., Loretelli, C. and Pandolfi, P. P. (2013) From pseudo-ceRNAs to circ-ceRNAs: a tale of cross-talk and competition. Nat. Struct. Mol. Biol., 20, 541-543

20. Cesana, M., Cacchiarelli, D., Legnini, I., Santini, T., Sthandier, O. Chinappi, M., Tramontano, A. and Bozzoni, I. (2011) A long noncoding RNA controls muscle differentiation by functioning as a competing endogenous RNA. Cell, 147, 358-369

21. Karreth, F. A., Tay, Y., Perna, D., Ala, U., Tan, S. M., Rust, A. G., DeNicola, G., Webster, K. A., Weiss, D., Perez-Mancera, P. A., et al. (2011) In vivo identification of tumor- suppressive PTEN ceRNAs in an oncogenic BRAF-induced mouse model of melanoma. Cell, 147, 382 395

22. Sumazin, P., Yang, X., Chiu, H. S., Chung, W. J., Iyer, A., LlobetNavas, D., Rajbhandari, P., Bansal, M., Guarnieri, P., Silva, J., et al. (2011) An extensive microRNA-mediated network of RNA-RNA interactions regulates established oncogenic pathways in glioblastoma. Cell, 147, 370-381

23. Tay, Y., Kats, L., Salmena, L., Weiss, D., Tan, S. M., Ala, U., Karreth,
F., Poliseno, L., Provero, P., Di Cunto, F., et al. (2011) Codingindependent regulation of the tumor suppressor PTEN by competing endogenous mRNAs. Cell, 147, 344-357

24. Memczak, S., Jens, M., Elefsinioti, A., Torti, F., Krueger, J., Rybak, A., Maier, L., Mackowiak, S. D., Gregersen, L. H., Munschauer, M., et al. (2013) Circular RNAs are a large class of animal RNAs with regulatory potency. Nature, 495, 333-338

25. Kumar, M. S., Armenteros-Monterroso, E., East, P., Chakravorty, P., Matthews, N., Winslow, M. M. and Downward, J. (2014) HMGA2 functions as a competing endogenous RNA to promote lung cancer progression. Nature, 505, 212-217

26. Peng, H., Lu, M. and Selaru, F. M. (2015) The genome-wide gene expression profiling to predict competitive endogenous RNA network in hepatocellular cancer. Genom Data, 4, 93-95

27. Qi, X., Zhang, D. H., Wu, N., Xiao, J. H., Wang, X. and Ma, W. (2015) ceRNA in cancer: possible functions and clinical implications. J. Med. Genet., 52, 710-718

28. Zhang, J., Fan, D., Jian, Z., Chen, G. G. and Lai, P. B. (2015) Cancer specific long noncoding RNAs show differential expression patterns and competing endogenous RNA potential in hepatocellular carcinoma. PLoS One, 10, e0141042

29. Bosson, A. D., Zamudio, J. R. and Sharp, P. A. (2014) Endogenous miRNA and target concentrations determine susceptibility to potential ceRNA competition. Mol. Cell, 56, 347-359

30. Mayya, V. K. and Duchaine, T. F. (2015) On the availability of microRNA-induced silencing complexes, saturation of microRNAbinding sites and stoichiometry. Nucleic Acids Res., 43, 7556-7565

31. Yuan, Y., Liu, B., Xie, P., Zhang, M. Q., Li, Y., Xie, Z. and Wang, X. (2015) Model-guided quantitative analysis of microRNA-mediated regulation on competing endogenous RNAs using a synthetic gene circuit. Proc. Natl. Acad. Sci. USA, 112, 3158-3163

32. Denzler, R., Agarwal, V., Stefano, J., Bartel, D. P. and Stoffel, M. (2014) Assessing the ceRNA hypothesis with quantitative measurements of miRNA and target abundance. Mol. Cell, 54, 766-776

33. Hausser, J. and Zavolan, M. (2014) Identification and consequences of miRNA-target interactions - beyond repression of gene expression. Nat. Rev. Genet., 15, 599-612

34. Lim, L. P., Lau, N. C., Garrett-Engele, P., Grimson, A., Schelter, J. M., Castle, J., Bartel, D. P., Linsley, P. S. and Johnson, J. M. (2005) Microarray analysis shows that some microRNAs downregulate large numbers of target mRNAs. Nature, 433, 769-773

35. Kou, Y., Qiao, L. and Wang, Q. (2015) Identification of core miRNA based on small RNA-seq and RNA-seq for colorectal cancer by bioinformatics. Tumour Biol., 36, 2249-2255

36. Wang, Z., Gerstein, M. and Snyder, M. (2009) RNA-Seq: a revolutionary tool for transcriptomics. Nat. Rev. Genet., 10, 57-63

37. Bazzini, A. A., Lee, M. T. and Giraldez, A. J. (2012) Ribosome profiling shows that miR-430 reduces translation before causing mRNA decay in zebrafish. Science, 336, 233-237

38. Baek, D., Villén, J., Shin, C., Camargo, F. D., Gygi, S. P. and Bartel, D. P. (2008) The impact of microRNAs on protein output. Nature, 455, 64 71

39. Hausser, J., Syed, A. P., Selevsek, N., van Nimwegen, E., Jaskiewicz, L., Aebersold, R. and Zavolan, M. (2013) Timescales and bottlenecks in miRNA-dependent gene regulation. Mol. Syst. Biol., 9, 711

40. Selbach, M., Schwanhäusser, B., Thierfelder, N., Fang, Z., Khanin, R and Rajewsky, N. (2008) Widespread changes in protein synthesis induced by microRNAs. Nature, $455,58-63$ 
41. Broughton, J. P. and Pasquinelli, A. E. (2013) Identifying Argonaute binding sites in Caenorhabditis elegans using iCLIP. Methods, 63, 119-125

42. Chi, S. W., Zang, J. B., Mele, A. and Darnell, R. B. (2009) Argonaute HITS-CLIP decodes microRNA-mRNA interaction maps. Nature, 460, 479-486

43. Konig, J., Zarnack, K., Rot, G., Curk, T., Kayikci, M., Zupan, B., Turner, D. J., Luscombe, N. M. and Ule, J. (2011) iCLIP-transcriptome-wide mapping of protein-RNA interactions with individual nucleotide resolution. J. Vis. Exp., e2638

44. Loeb, G. B., Khan, A. A., Canner, D., Hiatt, J. B., Shendure, J., Darnell, R. B., Leslie, C. S. and Rudensky, A. Y. (2012) Transcriptome-wide miR-155 binding map reveals widespread noncanonical microRNA targeting. Mol. Cell, 48, 760-770

45. Sutandy, F. X., Hildebrandt, A. and König, J. (2016) Profiling the binding sites of RNA-binding proteins with nucleotide resolution using iCLIP. Methods Mol. Biol., 1358, 175-195

46. Zisoulis, D. G., Lovci, M. T., Wilbert, M. L., Hutt, K. R., Liang, T. Y., Pasquinelli, A. E. and Yeo, G. W. (2010) Comprehensive discovery of endogenous Argonaute binding sites in Caenorhabditis elegans. Nat. Struct. Mol. Biol., 17, 173-179

47. Kishore, S., Jaskiewicz, L., Burger, L., Hausser, J., Khorshid, M. and Zavolan, M. (2011) A quantitative analysis of CLIP methods for identifying binding sites of RNA-binding proteins. Nat. Methods, 8, 559-564

48. Zhang, C. and Darnell, R. B. (2011) Mapping in vivo protein-RNA interactions at single-nucleotide resolution from HITS-CLIP data. Nat. Biotechnol., 29, 607-614

49. Levine, E., Zhang, Z., Kuhlman, T. and Hwa, T. (2007) Quantitative characteristics of gene regulation by small RNA. PLoS Biol., 5, e229.

50. Mukherji, S., Ebert, M. S., Zheng, G. X., Tsang, J. S., Sharp, P. A. and van Oudenaarden, A. (2011) MicroRNAs can generate thresholds in target gene expression. Nat. Genet., 43, 854-859

51. Schmiedel, J. M., Klemm, S. L., Zheng, Y., Sahay, A., Blüthgen, N., Marks, D. S. and van Oudenaarden, A. (2015) MicroRNA control of protein expression noise. Science, 348, 128-132

52. Milo, R., Jorgensen, P., Moran, U., Weber, G. and Springer, M. (2010) BioNumbers - the database of key numbers in molecular and cell biology. Nucleic Acids Res., 38, D750-D753

53. Schwanhäusser, B., Busse, D., Li, N., Dittmar, G., Schuchhardt, J., Wolf, J., Chen, W. and Selbach, M. (2011) Global quantification of mammalian gene expression control. Nature, 473, 337-342

54. Buchler, N. E. and Louis, M. (2008) Molecular titration and ultrasensitivity in regulatory networks. J. Mol. Biol., 384, 1106-1119

55. Ala, U., Karreth, F. A., Bosia, C., Pagnani, A., Taulli, R., Léopold, V., Tay, Y., Provero, P., Zecchina, R. and Pandolfi, P. P. (2013) Integrated transcriptional and competitive endogenous RNA networks are crossregulated in permissive molecular environments. Proc. Natl. Acad. Sci. USA, 110, 7154-7159

56. Martinez, N. J. and Gregory, R. I. (2013) Argonaute2 expression is post-transcriptionally coupled to microRNA abundance. RNA, 19, 605-612

57. Wang, D., Zhang, Z., O’Loughlin, E., Lee, T., Houel, S., O'Carroll, D., Tarakhovsky, A., Ahn, N. G. and Yi, R. (2012) Quantitative functions of Argonaute proteins in mammalian development. Genes Dev., 26, 693-704

58. Riba, A., Bosia, C., El Baroudi, M., Ollino, L. and Caselle, M. (2014) A combination of transcriptional and microRNA regulation improves the stability of the relative concentrations of target genes. PLoS Comput. Biol., 10, e1003490

59. Poliseno, L., Salmena, L., Zhang, J., Carver, B., Haveman, W. J. and Pandolfi, P. P. (2010) A coding-independent function of gene and pseudogene mRNAs regulates tumour biology. Nature, 465, 10331038

60. Figliuzzi, M., Marinari, E. and De Martino, A. (2013) MicroRNAs as a selective channel of communication between competing RNAs: a steady-state theory. Biophys. J., 104, 1203-1213

61. Nitzan, M., Steiman-Shimony, A., Altuvia, Y., Biham, O. and Margalit, H. (2014) Interactions between distant ceRNAs in regulatory networks. Biophys. J., 106, 2254-2266

62. Noorbakhsh, J., Lang, A. H. and Mehta, P. (2013) Intrinsic noise of microRNA-regulated genes and the ceRNA hypothesis. PLoS One, 8, e72676

63. Wang, P., Zhi, H., Zhang, Y., Liu, Y., Zhang, J., Gao, Y., Guo, M., Ning, S. and Li, X. (2015) miRSponge: a manually curated database for experimentally supported miRNA sponges and ceRNAs. Database (Oxford), 2015, 2015

64. Yu, G., Yao, W., Gumireddy, K., Li, A., Wang, J., Xiao, W., Chen, K., Xiao, H., Li, H., Tang, K., et al. (2014) Pseudogene PTENPI functions as a competing endogenous RNA to suppress clear-cell renal cell carcinoma progression. Mol. Cancer Ther., 13, 3086-3097

65. Jeyapalan, Z., Deng, Z., Shatseva, T., Fang, L., He, C. and Yang, B. B. (2011) Expression of CD44 3'-untranslated region regulates endogenous microRNA functions in tumorigenesis and angiogenesis. Nucleic Acids Res., 39, 3026-3041

66. Rutnam, Z. J. and Yang, B. B. (2012) The non-coding 3' UTR of CD44 induces metastasis by regulating extracellular matrix functions. J. Cell. Sci., 125, 2075-2085

67. Xia, T., Chen, S., Jiang, Z., Shao, Y., Jiang, X., Li, P., Xiao, B. and Guo, J. (2015) Long noncoding RNA FER1L4 suppresses cancer cell growth by acting as a competing endogenous RNA and regulating PTEN expression. Sci Rep, 5, 13445

68. Jackson, A. L., Burchard, J., Schelter, J., Chau, B. N., Cleary, M., Lim, L. and Linsley, P. S. (2006) Widespread siRNA "off-target" transcript silencing mediated by seed region sequence complementarity. RNA, 12, 1179-1187

69. Schafer, S., Adami, E., Heinig, M., Rodrigues, K. E., Kreuchwig, F., Silhavy, J., van Heesch, S., Simaite, D., Rajewsky, N., Cuppen, E., et al. (2015) Translational regulation shapes the molecular landscape of complex disease phenotypes. Nat Commun, 6, 7200

70. Jiang, J., Wakimoto, H., Seidman, J. G. and Seidman, C. E. (2013) Allele-specific silencing of mutant Myh6 transcripts in mice suppresses hypertrophic cardiomyopathy. Science, 342, 111-114

71. Chiu, H. S., Llobet-Navas, D., Yang, X., Chung, W. J., AmbesiImpiombato, A., Iyer, A., Kim, H. R., Seviour, E. G., Luo, Z., Sehgal, V., et al. (2015) Cupid: simultaneous reconstruction of microRNAtarget and ceRNA networks. Genome Res., 25, 257-267

72. Tan, J. Y., Sirey, T., Honti, F., Graham, B., Piovesan, A., Merkenschlager, M., Webber, C., Ponting, C. P. and Marques, A. C. (2015) Extensive microRNA-mediated crosstalk between lncRNAs and mRNAs in mouse embryonic stem cells. Genome Res., 25, 655-666

73. Xu, J., Li, Y., Lu, J., Pan, T., Ding, N., Wang, Z., Shao, T., Zhang, J., Wang, L. and Li, X. (2015) The mRNA related ceRNA-ceRNA landscape and significance across 20 major cancer types. Nucleic Acids Res., 43, 8169-8182 